\title{
CAR T-Cell therapies in lymphoma: current landscape, ongoing investigations, and future directions
}

\author{
J. Erika Haydu, Jeremy S. Abramson \\ Center for Lymphoma, Massachusetts General Hospital \& Harvard Medical School, Boston, MA 02114, USA.
}

Correspondence to: Jeremy S. Abramson, Director, Center for Lymphoma, Massachusetts General Hospital \& Harvard Medical School, 55 Fruit Street, Boston, MA 02114, USA. E-mail: jabramson@mgh.harvard.edu

How to cite this article: Haydu JE, Abramson JS. CAR T-Cell therapies in lymphoma: current landscape, ongoing investigations, and future directions. J Cancer Metastasis Treat 2021;7:36. https://dx.doi.org/10.20517/2394-4722.2021.39

Received: 16 Feb 2021 First Decision: 8 May 2021 Revised: 14 May 2021 Accepted: 24 May 2021 First online: 25 May 2021

Academic Editors: Francesca Montanari, Lucio Miele Copy Editor: Xi-Jun Chen Production Editor: Xi-Jun Chen

\begin{abstract}
Chimeric antigen receptor (CAR) T-cell therapy has significantly improved outcomes for patients with relapsed/refractory large B-cell lymphoma, mantle cell lymphoma, and follicular lymphoma, with multiple FDAapproved CAR T products now commercially available. Ongoing studies seek to move CAR T-cells to earlier lines of therapy and to characterize the efficacy and safety of CAR T-cell approaches in additional lymphoma histologies including relapsed/refractory follicular lymphoma and chronic lymphocytic leukemia. Other areas of active research address CAR T in combination with other lymphoma-directed therapies, and mechanisms of CAR T resistance. This review focuses on the FDA-approved anti-CD19 CAR T products for B-cell lymphomas, management of CAR T-cell-associated toxicities, approaches to bridging therapy, and ongoing clinical trials and future research directions across a broad range of lymphoma histologies.
\end{abstract}

Keywords: CAR T-cells, cell therapy, adoptive immunotherapy, non-Hodgkin lymphoma, aggressive lymphoma, indolent lymphoma, mantle cell lymphoma, diffuse large B-cell lymphoma

\section{INTRODUCTION}

Non-Hodgkin lymphomas (NHL) comprise a heterogenous group of neoplasms arising from B- or Tlymphocytes, with 81,560 new cases and 20,720 deaths from NHL estimated in the United States in $2021^{[1]}$. 
NHLs range from highly aggressive malignancies which are typically fatal without treatment to indolent diseases, and thus treatment approaches vary based on histology and clinical presentation ${ }^{[2]}$.

The most common NHL is diffuse large B-cell lymphoma (DLBCL), an aggressive lymphoma that can be cured with standard frontline chemoimmunotherapy in $60 \%-70 \%$ of patients but with historically poor outcomes for relapsed/refractory disease. Patients with relapsed DLBCL after autologous stem cell transplant (ASCT) or with chemotherapy-refractory disease have a particularly dismal prognosis, with a median overall survival (OS) of only 6 month $\mathrm{s}^{[3]}$. Mantle cell lymphoma (MCL) represents approximately $6 \%$ of all newly diagnosed NHLs in the United States and is typically responsive to frontline chemoimmunotherapy but invariably recurs and has a median OS of approximately 10 years. Outcomes are worse for MCL patients with disease progression after Bruton's tyrosine kinase inhibition (BTKi), with a median survival of only 3-11 months in this setting ${ }^{[4-6]}$. Follicular lymphoma (FL) and marginal zone lymphoma (MZL) are characteristically indolent B-cell lymphomas that are highly responsive to upfront chemoimmunotherapy strategies and may not limit life expectancy, but none the less remain incurable in the majority of cases. FL and MZL patients who relapse within 2 years of initial therapy have a much worse prognosis, with a median OS of only 5 years $^{[7]}$. Chronic lymphocytic leukemia (CLL)/small lymphocytic leukemia (SLL) is an indolent B-cell lymphoma which is highly treatable with targeted agents yet remains incurable, and tumors with certain high-risk molecular features, including 17p deletion or TP53 mutations, which progress after BTKi and/or BCL2-directed approaches confer an inferior prognosis ${ }^{[8]}$. Thus, there is a critical need for additional treatment options for patients across the spectrum of B-cell lymphomas, and chimeric antigen receptor (CAR) T-cell therapy is poised to help fill this need.

\section{Biology of CAR T-cells}

Currently available CAR T-cells are generated by transducing a patient's autologous T-cells with inactivated viral vectors (lentiviral or retroviral constructs) to produce T-cells capable of expressing an antigen receptor targeted to a tumor cell surface antigen, along with a transmembrane spacer, a co-stimulatory domain (usually CD28 or $4-1 \mathrm{BB}$ ), and an intracellular signaling domain (CD3z) to lead to T-cell activation. CAR T approaches allow for MHC-independent recognition of tumor cells, thereby conferring the specificity of a monoclonal antibody to a T-cell. The most widely studied CAR T products now FDA-approved in aggressive large $\mathrm{B}$-cell lymphomas ${ }^{[9-11]}, \mathrm{MCL}^{[12]}$, and $\mathrm{FL}^{[13]}$ target the $\mathrm{CD} 19$ antigen, which is broadly expressed on mature B-cell lymphomas as well as on B-cell acute lymphoblastic leukemia. Full activation of a T-cell requires both a primary signal through the TCR as well as co-stimulation to avoid anergy ${ }^{[14]}$, and incorporation of co-stimulatory domains, which were absent in first generation CARs, has led to improvements in clinical efficacy ${ }^{[15]}$. The type of co-stimulatory domain leads to differences in CAR T-cell dynamics, with CD28 co-stimulation resulting in quicker and higher peak CAR T expansion compared to 4$1 \mathrm{BB}-\mathrm{co}-$ stimulation $^{[16]}$, and is associated with differences in toxicity profiles (see below).

After a patient's T-cells are collected via apheresis, the manufacturing process typically takes 3-4 weeks. Patients may or may not receive "bridging" chemotherapy to control their underlying malignancy while awaiting receipt of their genetically modified CAR T-cells. Prior to CAR T-cell infusion, patients receive lymphodepleting chemotherapy which has been shown to promote proliferation and expansion of the T-cell product and to prevent an immune response directed against the CAR T-cells ${ }^{[17]}$. Patients are subsequently monitored for potential toxicities.

\section{APPROVED CAR T-CELL PRODUCTS DLBCL and other large B-cell lymphomas}

There are now three FDA-approved anti-CD19 CAR T-cell products for relapsed/refractory DLBCL and 
other aggressive large B-cell lymphomas: axicabtagene ciloleucel (axi-cel), tisagenlecleucel (tisa-cel), and lisocabtagene maraleucel (liso-cel) [Table 1]. These three CAR T-cell products were studied in separate clinical trials ${ }^{[9-11]}$, which differ in construct design, manufacturing process, and side effect profiles but in general are all capable of producing durable remissions in a significant proportion of patients.

Axi-cel (Yescarta) was the first anti-CD19 CAR T product approved by the FDA (2017) and the European Medicines Agency (EMA) (2018) for DLBCL. Axi-cel is generated by transfecting bulk T-cells using a retroviral vector and includes a CD28 co-stimulatory domain. ZUMA-1 was the pivotal single-arm phase 2 clinical trial of axi-cel in patients with DLBCL, primary mediastinal B-cell lymphoma (PMBCL), high grade B-cell lymphoma (HGBCL) with double-hit cytogenetics, and transformed follicular lymphoma (tFL $)^{[10]}$. Eligible patients had disease progression after at least two prior lines of therapy and were considered chemotherapy-refractory, defined as progression or stable disease as best response to the most recent prior treatment, or relapse within one year from ASCT. Bridging chemotherapy was not permitted and lymphodepletion was performed with three days of fludarabine and cyclophosphamide. CAR T product was successfully manufactured for 110 patients and ultimately infused in 101/111 enrolled patients. In a recently updated analysis, the objective response rate (ORR) was $82 \%$ with a complete response (CR) in $58 \%$ of patients. The progression free survival (PFS) was 39\% and the median OS had not yet been reached at 27 months of median follow up ${ }^{[10,18]}$.

Tisa-cel (Kymriah) was the second anti-CD19 CAR T product to be FDA- and EMA-approved (2018) in DLBCL. Tisa-cel is generated from bulk T-cells with a lentiviral vector and incorporates a 4-1BB costimulatory domain. JULIET was the pivotal phase 2 clinical trial of tisa-cel in patients with relapsed/refractory DLBCL, double-hit lymphoma, or $\mathrm{tFL}^{[11]}$. Eligible patients had received at least two prior lines of chemotherapy but in contrast to the ZUMA-1 trial ${ }^{[10]}$, they were not required to be chemotherapyrefractory, although $55 \%$ of the JULIET patients were considered refractory and $49 \%$ had relapsed after ASCT. Bridging chemotherapy was allowed and was given to $92 \%$ of patients and lymphodepletion was performed with 3 days of fludarabine and cyclophosphamide or 2 days of bendamustine. Of the 165 patients enrolled in this study, 111 ultimately received CAR T-cells; in 12 instances, CAR T-cells were unable to be manufactured. The ORR with tisa-cel was 52\%, with a CR rate of $40 \%, 1$ year PFS of $35 \%$, and a 1 year OS of $49 \%$ for all treated patients (90\% among patients with a CR).

Liso-cel (Breyanzi) is the third major anti-CD19 CAR T product for relapsed/refractory DLBCL and was FDA-approved in $2021^{[9]}$. Similar to tisa-cel, liso-cel is manufactured using a lentiviral vector and a 4-1BB costimulatory domain but instead of transducing bulk T-cells, CD4+ and CD8+ T-cells are separated and transduced and then infused back into the patient at equal target doses ${ }^{[19]}$. This defined composition approach has been shown in preclinical data to improve CAR T-cell expansion and persistence and to optimize antitumor properties, though the relative importance in clinical trials has yet to be definitively established $^{[20]}$. TRANSCEND was the large seamless design clinical trial leading to the approval of liso-cel ${ }^{[9]}$. Eligible patients had relapsed/refractory large B-cell lymphomas (DLBCL, HGBCL with double- or triple-hit cytogenetics, and PMBCL), transformed indolent lymphoma (follicular, marginal zone, CLL/SLL, or lymphoplasmacytic), or grade 3B FL and had received a minimum of two prior therapies; prior stem cell transplant (autologous or allogeneic) was permitted. Patients with secondary CNS lymphoma were eligible for enrollment, in contrast to ZUMA- ${ }^{[10]}$ or JULIET ${ }^{[1]}$, which excluded patients with CNS disease. Bridging chemotherapy was allowed and was given to $59 \%$ of patients, lymphodepletion was performed with 3 days of fludarabine and cyclophosphamide, and CAR T-cells were given at three different dose levels. Of the 344 patients who underwent leukapheresis, 269 ultimately received liso-cel and an additional 25 received a nonconforming product (most commonly because one of the CD4 or CD8 cell components did not technically 
Table 1. Summary of anti-CD19 CAR T-cell products approved and under investigation in B-cell lymphomas

\begin{tabular}{|c|c|c|c|}
\hline Lymphoma & FDA-approved & $\begin{array}{l}\text { Additional histologies } \\
\text { included in pivotal trials }\end{array}$ & Highlighted ongoing studies \\
\hline \multicolumn{4}{|l|}{$\frac{\text { Large B-cell }}{(\mathrm{R} / \mathrm{R})}$} \\
\hline DLBCL & $\begin{array}{l}\text { Axi-cel (ZUMA-1) } \\
\text { Tisa-cel (JULIET) } \\
\text { Liso-cel (TRANSCEND) }^{[11]}\end{array}$ & & $\begin{array}{l}\text { 1st line (high-risk): Axi-cel (ZUMA-12) })^{[37]} \\
\text { 2nd line: Axi-cel (ZUMA-7 })^{[35]} \text {, Tisa-cel (BELINDA) }{ }^{[36]} \text {, Liso-cel } \\
\text { (TRANSFORM) } \\
\text { CAR T plus (R/R): Axi-cel (ZUMA-6; atezolizumab) })^{[54]} \text {, Tisa-cel } \\
\text { (PORTIA; pembrolizumab) }^{[55]} \text {, Tisa-cel (NCTO3876028; ibrutinib), } \\
\text { Liso-cel (PLATFORM; durvalumab, CC-122/220) } \\
\text { Allogeneic CAR (R/R): ALLO-501A (ALPHA-2), CAR-NK } \\
\text { (NCT03056339) }^{[62]}\end{array}$ \\
\hline $\mathrm{HGBCL}$ & $\begin{array}{l}\text { Axi-cel (ZUMA-1) })^{[10]} \\
\text { Tisa-cel (JULIET) } \\
\text { Liso-cel (TRANSCEND) }^{[1]]}\end{array}$ & & \\
\hline PMBCL & $\begin{array}{l}\text { Axi-cel (ZUMA-1) })^{[10]} \\
\text { Liso-cel (TRANSCEND) }\end{array}$ & & \\
\hline \multicolumn{4}{|l|}{$\underline{\text { Transformed }}$} \\
\hline $\mathrm{FL}$ & $\begin{array}{l}\text { Axi-cel (ZUMA-1) })^{[10]} \\
\text { Tisa-cel (JULIET) } \\
\text { Liso-cel (TRANSCEND) }^{[1]]}\end{array}$ & & \\
\hline MZL & Liso-cel (TRANSCEND) $)^{[9]}$ & & \\
\hline $\mathrm{CLL} / \mathrm{SLL}$ & Liso-cel (TRANSCEND) $)^{[9]}$ & & \\
\hline Overall & $\begin{array}{l}\text { Axi-cel }(Z U M A-1)^{[10,18]} \\
\text { ORR }=82 \%, C R R=58 \% \\
\text { Tisa-cel }(J U L I E T)^{[11]} \\
\text { ORR }=52 \%, C R R=40 \% \\
\text { Liso-cel }(\text { TRANSCEND) } \\
\text { ORR }=73 \%, \text { CRR }=53 \%\end{array}$ & & \\
\hline$M C L(R / R)$ & $\begin{array}{l}\text { Brexu-cel }(\text { ZUMA-2 })^{[12]} \\
\text { ORR }=93 \%, \text { CRR }=67 \%\end{array}$ & $\begin{array}{l}\text { Liso-cel }(\text { TRANSCEND })^{[25]} \\
\text { ORR }=84 \%, \text { CRR }=59 \%\end{array}$ & \\
\hline$F L(R / R)$ & $\begin{array}{l}\text { Axi-cel }(\text { ZUMA-5 })^{[13]} \\
\text { ORR }=94 \%, C R R=80 \%\end{array}$ & & $\begin{array}{l}\text { Tisa-cel }\left(\text { Elara }{ }^{[39]}\right. \\
\text { ORR }=83 \%, 65 \%\end{array}$ \\
\hline FL Grade 3B & Liso-cel (TRANSCEND) $)^{[9]}$ & & \\
\hline$M Z L(R / R)$ & & & $\begin{array}{l}\text { Axi-cel }(Z U M A-5)^{[13]} \\
\text { ORR }=85 \%, C R R=60 \%\end{array}$ \\
\hline $\begin{array}{l}\mathrm{CLL} / \mathrm{SLL} \\
(\mathrm{R} / \mathrm{R})\end{array}$ & & & $\begin{array}{l}\text { Liso-cel }(\text { TRANSCEND })^{[43]} \\
\text { ORR }=82 \%, \text { CRR }=45 \% \\
\left.\text { CAR T plus BTKi: Liso-cel (PLATFORM; TRANSCEND }{ }^{[59]}\right), \text { CD19 CAR } \\
(\text { NCT01865617) })^{[58]}\end{array}$ \\
\hline PCNSL & & & Tisa-cel (NCT04134117), City of Hope ${ }^{[53]}$ \\
\hline
\end{tabular}

DLBCL: Diffuse large B-cell lymphoma; R/R: relapsed/refractory; HGBCL: high-grade B-cell lymphoma; PMBC: primary mediastinal B-cell lymphoma; FL: follicular lymphoma; MZL: marginal zone lymphoma; CLL: chronic lymphocytic leukemia; SLL: small lymphocytic leukemia; MCL: mantle cell lymphoma; PCNSL: primary CNS Iymphoma; ORR: objective/overall response rate; CRR: complete response rate; SOC: standard of care.

meet the requirements to be considered liso-cel). Product could not be manufactured for 2 patients and 48 patients had lymphoma complications or died before infusion, reflecting the high-risk nature of this population. Ultimately 256 patients were included in the efficacy-evaluable set. The ORR was $73 \%$, with a CR rate of $53 \%$ and an estimated PFS and OS at 1 year of $44 \%$ and $58 \%$, respectively, for the entire population. Among complete responders, $86 \%$ were alive at 1 year. Liso-cel is the only CAR T-cell product for large B-cell lymphomas that was studied in certain high-risk patient populations including patients with secondary CNS disease, prior allogeneic stem cell transplant, ECOG performance status of 2, and in certain histologies such as DLBCL transformed from indolent lymphomas other than FL (CLL, marginal zone, Waldenstroms) and grade $3 \mathrm{~B} \mathrm{FL}$, which is thus reflected in the broader range of eligible histologies included in the FDA label. 
Axi-cel, tisa-cel, and liso-cel have not been compared head-to-head, and probably never will be, but since all have been shown to produce durable remissions in a significant proportion of treated patients, choice of product for an individual patient may take into account additional factors including availability, processing time, and side effect profiles (discussed below). Real-world experience with axi-cel and tisa-cel shows that up to $62 \%$ of "real world" patients treated with a commercial product would not have met clinical trial eligibility criteria, but encouragingly, outcomes are similar for these patients compared to patients treated on the pivotal trials ${ }^{[21-24]}$.

\section{Mantle cell lymphoma}

Recently, the anti-CD19 CAR T product brexucabtagene autoleucel (brexu-cel, previously KTE-X19) was FDA-approved (2020) for relapsed or refractory MCL [Table 1] based on the results from the phase 2 openlabel multicenter ZUMA-2 trial ${ }^{[12]}$. Eligible patients were previously treated with anthracycline or bendamustine, anti-CD20 monoclonal antibody, and BTKi (ibrutinib or acalabrutinib), and had disease that was relapsed or considered refractory to up to five previous regimens. Although brexu-cel shares a CD28 costimulatory domain with axi-cel, the brexu-cel manufacturing process is unique from other FDAapproved CAR T-cell products because circulating CD19-expressing malignant B-cells are removed during production to help reduce tumor cell contamination and possible CAR T-cell activation and exhaustion. Bridging therapy was permitted with investigator's choice of steroid, BTKi (ibrutinib or acalabrutinib), or steroid plus BTKi, and patients underwent lymphodepleting chemotherapy with fludarabine and cyclophosphamide. Overall, brexu-cel was successfully manufactured for $71 / 74$ patients and administered to 68 patients. Of the 60 treated patients with at least 7 months of follow-up, 93\% responded, $67 \%$ completely. At the time of data cutoff, a total of $57 \%$ of patients in the primary efficacy analysis and $78 \%$ of patients with a CR remained in remission. The estimated 1 -year PFS was $61 \%$ and the OS was $83 \%$.

Liso-cel has also been studied in relapsed/refractory MCL, and an interim analysis of this cohort from the TRANSCEND trial has been presented ${ }^{[25]}$. Eligible patients had relapsed/refractory MCL after at least one prior line of therapy and were treated with liso-cel at two different doses following lymphodepletion and optional bridging therapy. At the time of data cutoff, 32 patients had received liso-cel [the majority of patients (26/32) at the higher CAR+ T-cell dose]. Patients had received a median of three prior therapies, including 28 patients (88\%) who had received prior BTKi therapy and 11 patients (34\%) considered BTKirefractory. Of the 32 patients, the ORR was $84 \%$, with $59 \%$ of patients achieving a CR, and $74 \%$ of the 27 responders had an ongoing response at the time of analysis.

\section{Follicular lymphoma}

Follicular lympoma is an indolent disease with a favorable prognosis in the majority of patients; however, FL which is refractory to both alkylating agents and rituximab, and FL that progresses within 24 months of initial chemoimmunotherapy, are associated inferior $\mathrm{OS}^{[7]}$. Initial studies of anti-CD19 CAR T approaches in relapsed/refractory FL have demonstrated promising efficacy with CR rates ranging from $71 \%-88 \%{ }^{[13,26,27]}$. ZUMA-5 is the largest CAR T study to date in relapsed/refractory indolent NHL ${ }^{[13]}$. In ZUMA-5, 146 patients received axi-cel (124 FL and $22 \mathrm{MZL}$ patients). At a median follow-up of 17.5 months, the ORR was $94 \%$ for FL and $85 \%$ for MZL, with CR rates of $80 \%$ and $60 \%$, respectively, and ongoing responses in $62 \%$ of all patients. This study has led to the recent FDA approval (2021) of axi-cel in relapsed/refractory FL [Table 1].

\section{Managing CAR T-cell toxicities}

The antigen targeting and immune activation that make CD19 CAR T-cells highly effective at eradicating tumor cells are also responsible for the potential toxicities, which include cytokine release syndrome (CRS) and a neurologic toxicity syndrome [immune effector cell associated neurologic syndrome (ICANS)]. The 
incidence and severity of CRS and ICANS vary based on CAR T-cell product but tend to follow a predictable course and are relatively manageable and reversible. The choice of co-stimulatory domain is an important predictor of toxicity, with $\mathrm{CD} 28$ co-stimulation leading to a more rapid and higher peak expansion of CAR T-cells compared to the more gradual expansion and longer persistence of T-cells engineered with $4-1 \mathrm{BB}$ co-stimulation ${ }^{[16]}$. In practice, the kinetics of $\mathrm{CD} 28$ co-stimulation appear to result in a quicker onset and increased incidence and higher severity of toxicity compared to a 4-1BB co-stimulated product. High tumor burden has also been shown to be predictive of severe $\mathrm{CRS}^{[28]}$ and neurotoxicity ${ }^{[23]}$.

\section{Cytokine release syndrome}

Cytokine release syndrome is characterized by the presence of fever and in more severe cases can progress to hypotension, hypoxia, capillary leak, organ injury, and delirium. Manifestations of CRS can affect almost any organ system. The incidence of CRS with the CD28 co-stimulated axi-cel in ZUMA-1 was 93\% for anygrade CRS and 13\% for severe (grade 3-4) CRS and occurred at a median of 2 days following CAR T-cell infusion $^{[10,18]}$. In the ZUMA-5 trial in indolent B-cell NHL, grade 3 or higher CRS occurred in $7 \%$ of axi-celtreated patients ${ }^{[13]}$. In JULIET, the incidence of CRS with the $4-1 \mathrm{BB}$ co-stimulated tisa-cel was $58 \%$ for anygrade and $22 \%$ for grades $3-4$, with a median onset at day $3^{[11]}$. The TRANSCEND trial with the $4-1 \mathrm{BB}$ costimulated liso-cel reported an incidence of any-grade CRS in $42 \%$ of patients with a median time to onset at day 5 , with only $2 \%$ of patients developing severe CRS ${ }^{[9]}$. In the relapsed/refractory MCL studies, $91 \%$ of patients treated with the CD28 co-stimulated brexu-cel in ZUMA-2 experienced CRS with grade 3 or higher in $15 \%$ of patients ${ }^{[12]}$, while in the TRANSCEND MCL cohort (4-1BB co-stimulated liso-cel), the overall CRS rate was $50 \%$, with only one grade 4 event and no grade 3 or 5 events ${ }^{[25]}$. Of note, CRS was graded using the Lee criteria $^{[29]}$ in ZUMA-1, ZUMA-2, ZUMA-5, and TRANSCEND and by the University of Pennsylvania scale in JULIET, which classifies more patients as grade 3 or 4 compared to Lee criteria. Consequently, the difference in grading systems complicates direct comparisons of grade 3-4 CRS rates, but there clearly does appear to be a lower CRS incidence and severity in patients treated with 4-1BB costimulated products.

CRS management includes supportive measures with intravenous fluids, antipyretics, and supplemental oxygen as needed. Treatment with the IL- 6 receptor antagonist tocilizumab can rapidly resolve fevers and vasopressor requirements in the majority of patients with severe CRS and is now FDA-approved for the treatment of grade 2-4 CRS. The rate of tocilizumab use on trial was $43 \%$ for patients treated with axi-cel in ZUMA-1, 15\% for patients treated with tisa-cel in JULIET, and $20 \%$ for patients treated with liso-cel in TRANSCEND ${ }^{[9-11]}$. In ZUMA-2, 59\% of MCL patients treated with brexu-cel received tocilizumab ${ }^{[12]}$. Corticosteroids can also ameliorate CRS and may be given concurrently with tocilizumab for grade 2-4 toxicity. Use of rescue medications for CRS has not been independently associated with impaired antitumor responses ${ }^{[10]}$.

\section{Neurologic toxicity}

Patients who develop ICANS with CAR T-cell therapy most commonly present with confusion but can also develop aphasia, somnolence, obtundation, seizures, and rarely cerebral edema. ICANS usually occurs in patients who have already had CRS and onsets as CRS is peaking or resolving; only rarely will ICANS occur in the absence of antecedent CRS. Neurologic toxicity has predominantly been graded with the Common Terminology Criteria for Adverse Events (CTCAE). In the 3 pivotal DLBCL CAR T trials, the incidence of neurotoxicity was $64 \%$ for axi-cel ( $28 \%$ of patients with grade $\geq 3$ ), $21 \%$ for tisa-cel ( $12 \%$ of patients with grade $\geq 3$ ), and $30 \%$ for liso-cel $(10 \% \text { of patients with grade } \geq 3)^{[9-11]}$. In ZUMA-5, grade 3 or higher neurologic events occurred in $19 \%$ of FL or MZL patients treated with axi-cel ${ }^{[13]}$. In the mantle cell CAR T trials, the incidence of neurologic events was $63 \%$ for patients treated with brexu-cel (31\% of patients with grade $\geq 3$ ) ${ }^{[12]}$ and $28 \%$ for patients treated with liso-cel (three grade 3 events and no grade 4 or 5 neurologic 
events $)^{[25]}$. Similar to CRS rates, the lower rates of neurologic toxicity with tisa-cel and liso-cel may reflect the kinetics associated with a 4-1BB co-stimulatory domain.

Neurologic toxicity is managed primarily with dexamethasone, which can improve symptoms and accelerate the rate of recovery from ICANS and has become the predominant treatment for high-grade neurotoxicity. Tocilizumab has shown only limited efficacy in treating ICANS ${ }^{[30-32]}$, perhaps because of poor CNS penetration ${ }^{[33]}$, and thus corticosteroids have remained the first line treatment for severe neurologic toxicity, with tocilizumab given only for patients with concurrent CRS and neurotoxicity. Patients at high risk of ICANS, or those with onset of ICANS symptoms, should also receive prophylactic antiepileptics such as levetiracetam. Rare cases of cerebral edema are typically treated with high dose methylprednisolone.

\section{Other CAR T-associated toxicities}

Cytopenias, B-cell aplasia, and hypogammaglobulinemia also occur following CAR T treatment. B-cell aplasia and hypogammaglobulinemia are attributed to on-target CD19 B-cell depletion, and notably most patients enrolling on CAR T-cell trials are hypogammaglobulinemic at baseline due to extensive prior lymphoma-directed therapies. Other cytopenias including neutropenia, lymphopenia, anemia, and thrombocytopenia may be secondary to lymphodepleting chemotherapy but have also been reported in the absence of conditioning and likely denote cytokine-mediated inflammatory marrow suppression ${ }^{[34]}$. Management of prolonged cytopenias is supportive until count recovery occurs. CD4 depletion may be prolonged and consideration should be given to pneumocystis and viral prophylaxis until sufficient CD4 recovery is established. Infusion of intravenous immunoglobulin post-CAR T-cell therapy may be required to prevent recurrent infections in the setting of hypogammaglobulinemia should patients experience severe or recurrent infectious episodes.

\section{ONGOING CAR T DEVELOPMENTS IN B-CELL NHLS DLBCL and other large B-cell lymphomas}

The pivotal trials of axi-cel, tisa-cel, and liso-cel enrolled relapsed/refractory patients treated with at least two prior lines of therapy. Thus, patients were generally heavily pre-treated with potentially less fit $\mathrm{T}$-cells from which to generate CAR T-cells. Moreover, a small proportion of patients in these trials never made it to CAR T-cell infusion due to progressive disease, reflective of the aggressive nature of these lymphomas. In this context, growing interest to assess the efficacy and safety of CAR T-cells in the second line has led to three large clinical trials comparing CAR T-cell therapy to ASCT at first relapse in DLBCL. ZUMA-7 $(\mathrm{NCT} 03391466)^{[35]}$, BELINDA (NCT03570892) $)^{[36]}$, and TRANSFORM (NCT03575351) are randomized, phase 3, open-label multicenter studies of anti-CD19 CAR T-cell therapy vs. standard of care (SOC) second line treatment in relapsed/refractory DLBCL or other aggressive B-cell NHLs initially treated with standard first line chemoimmunotherapy. In all three trials, patients are randomized 1:1 to CAR T-cell therapy or to SOC. Patients in the SOC arm receive standard second line salvage chemoimmunotherapy per the treating investigator's choice (e.g., R-ICE, R-DHAP, R-GDP) and if responding proceed with high-dose chemotherapy and ASCT. Crossover from SOC to CAR T is permitted for both BELINDA and TRANSFORM in patients not responding to second line chemotherapy. Interim results from these studies are eagerly anticipated.

Given that first line chemoimmunotherapy can cure a substantial fraction of patients with DLBCL, CAR Tcell therapy has been primarily studied in relapsed/refractory disease. However, there may be a population of patients at high risk of relapse who benefit from even earlier administration of CAR T-cells, although identifying these patients in the upfront setting can be challenging. ZUMA-12 is an ongoing phase 2, multicenter, open-label single arm study of axi-cel in patients with high risk large B-cell lymphoma who 
have a positive interim PET after 2 cycles of standard frontline chemoimmunotherapy ${ }^{[37]}$. For the 12 response-evaluable patients in a planned interim analysis, the ORR was $92 \%$ with a CR rate of $75 \%$, and $75 \%$ of patients had ongoing responses at data cut off. Interestingly, median peak CAR T-cell levels and cell expansion were higher in ZUMA-12 patients compared to ZUMA- ${ }^{[10]}$ patients, which may reflect improved CAR T health from exposure to fewer prior therapies. Although ZUMA-12 is not randomized and thus is susceptible to selection bias, and the value of early interim PET in predicting outcomes in DLBCL remains controversial although it may identify patients at increased risk of poorer outcomes ${ }^{[38]}$, the initial results from ZUMA-12 are promising and ultimately this and other studies, potentially with additional biomarkers beyond interim PET, may help identify patients who benefit from an early intensification approach.

\section{Follicular lymphoma and marginal zone lymphoma}

FL typically has an indolent course although a small subset of patients will transform to an aggressive large cell lymphoma which becomes life-threatening. Patients with tFL were eligible for the pivotal trials of CD19 CAR T-cells and response rates for tFL were generally on par with that seen in relapsed/refractory DLBCL. In the ZUMA-1 trial, 16 of the 101 axi-cel-treated patients had tFL and in an updated analysis, there was an ongoing response in $56 \%(9 / 16)$ of tFL patients at 24 months $^{[10,18]}$. The TRANSCEND large B-cell cohort included tFL (60/269; $22 \%$ of patients) as well as other transformed indolent lymphomas (iNHL) including MZL (18/296; 7\% of patients) ${ }^{[9]}$. The ORRs in the 57 evaluable tFL patients and the 18 evaluable transformed iNHL patients were $84.2 \%(48 / 57)$ and $61.1 \%(11 / 18)$, respectively. The TRANSCEND study also included a

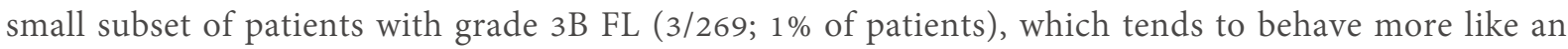
aggressive lymphoma; 2 of these patients went on to receive liso-cel and both remain in a CR at 1 year.

In addition to axi-cel, other CAR T-cell products are under investigation in relapsed/refractory FL. In a recently presented interim analysis of the ongoing phase 2 Elara trial of tisa-cel in relapsed/refractory FL, 52/97 tisa-cel treated patients were evaluable for efficacy, with an ORR of $83 \%$ in the ITT and $85 \%$ in the per protocol populations, with CR rates of $65 \%$ and $71 \%$, respectively ${ }^{[39]}$. Of the 97 Elara patients evaluable for safety, the rate of grade 3 or higher CRS or neurotoxicity was $0 \%$ and $2 \%$ respectively. ZUMA- $5^{[13]}$ and Elara $^{[39]}$ highlight the potential for CAR T therapy in relapsed/refractory FL and MZL and may ultimately lead to additional FDA approvals for these diseases.

\section{Chronic lymphocytic leukemia/small lymphocytic leukemia}

CAR T-cell therapy has also been investigated in patients with relapsed/refractory CLL/SLL. CLL/SLL is an indolent disease but patients with relapsed/refractory disease after BTKi and/or BCL2-directed therapy have a poor prognosis with limited treatment options. Results from small series of relapsed/refractory CLL patients treated with anti-CD19 CAR T-cell products have shown promising efficacy with tolerable side effect profiles. For example, all 4 CLL patients treated with a CD28 co-stimulated CAR T product had a response with 3 achieving a $\mathrm{CR}^{[40]}$, while response rates for patients treated with $4-1 \mathrm{BB}$ co-stimulated products in larger published series have ranged from $57 \%(8 / 14 ; 4 \mathrm{CR} \text { and } 4 \mathrm{PR})^{[41]}$ to $74 \%(14 / 19 ; 4 \mathrm{CR}$ and $10 \mathrm{PR})^{[42]}$.

Currently, several clinical trials are evaluating the safety and efficacy of anti-CD19 CAR T approaches in relapsed/refractory CLL. The ongoing, open-label, phase 1/2 TRANSCEND CLL 004 study (NCT03331198) reported interim results for patients treated with liso-cel in the phase 1 cohort $^{[43]}$. Eligible patients had received at least three prior regimens for standard risk disease or two prior regimens for high risk disease, with high risk factors defined as 17p deletion, TP53 mutation, unmutated IGHV, or complex karyotype. All patients had to have failed or been intolerant to a BTKi. The interim analysis included 23 safety-evaluable and 22 efficacy-evaluable patients, and within this group, $83 \%$ of patients had high risk disease and all had received prior BTKi. The ORR was $82 \%(18 / 22)$ with a CRR of $45 \%(10 / 22)$. At a median follow up of 18 
months, the median duration of response was not reached, and the median PFS was 18 months. Safety profiles were similar to what has been seen previously with liso-cel. Interestingly, of the 20 patients evaluable for minimal residual disease (MRD), 75\% (15/20) had undetectable MRD in the blood and 87\% (13/15) also had undetectable MRD in the bone marrow, raising the possibility of CLL/SLL disease eradication with CAR T approaches.

\section{Bridging patients to CAR T-cell therapies}

Bridging patients to CAR T therapy is an area of unmet clinical need. Bridging was permitted in the JULIET $^{[11]}$ and TRANSCEND ${ }^{[9]}$ trials of relapsed/refectory large B-cell lymphomas, with the majority of patients receiving bridging therapy (92\% and $72 \%$, respectively). ZUMA- ${ }^{[10]}$ prohibited bridging but most patients receiving axi-cel in the real world context have also required bridging therapy ${ }^{[44]}$. Importantly, bridging therapy has been associated with lower response rates and durability, with an ORR of $67.3 \%$ for bridged patients in TRANSCEND compared to $80.2 \%$ for non-bridged patients, as well as a higher incidence of CRS and neurologic events in bridged patients ${ }^{[9]}$. A retrospective analysis of 298 patients with large B-cell lymphoma treated with commercially available axi-cel found that bridging therapy was associated with an inferior $\mathrm{OS}\left(\mathrm{HR}=1.7\right.$ in bridged $v$ s. non bridged patients ${ }^{[23]}$. The inferior outcomes associated with bridging are likely multifactorial and may largely reflect the characteristics of patients who require bridging therapy: patients with bulkier, more rapidly progressive, and/or symptomatic disease who are likely to have additional risk factors for inferior outcomes and increased toxicities, including high tumor burden.

Current bridging approaches are not effective in many patients, a perhaps unsurprising observation given that patients eligible for CAR T therapy have typically had chemotherapy-refractory disease. Importantly, a significant subset of patients never make it to CAR T-cell infusion in part due to failure of bridging therapy to control disease, with 54/165 patients in JULIET ultimately not receiving CAR T-cells ${ }^{[26]}$ and death from disease progression in 33/344 patients in TRANSCEND ${ }^{[9]}$. In the brexu-cel relapsed/refractory MCL trial, the majority of the bridged patients actually had an increase in median tumor burden by radiographic imaging over the bridging period ${ }^{[12]}$, further reflecting the challenging nature of controlling relapsed/refractory disease with current bridging techniques and underscoring the need for novel approaches that maximize efficacy and limit systemic toxicity.

In DLBCL patients awaiting CAR $\mathrm{T}$ therapy, bridging has typically been attempted with steroids, chemoimmunotherapy, and/or radiation. Steroids are a viable bridging option with less systemic toxicity than chemoimmunotherapy, though responses are often short lived. Radiation is an attractive bridging modality for localized and/or focally symptomatic disease given the minimal systemic toxicity. Potential novel bridging approaches include agents which have demonstrated efficacy in relapsed/refractory DLBCL, including brentuximab vedotin for $\mathrm{CD} 30+$ diseas $^{[45]}$, lenalidomide or BTKi for non-GCB disease $\mathrm{e}^{[46]}$, and the CD79B antibody drug conjugate polatuzumab vedotin ${ }^{[47]}$. The anti-CD19 therapy tafastimab has promising activity in combination with lenalidomide in relapsed/refractory DLBCL for which it is FDA approved ${ }^{[48]}$, but the impact of CD19-targeted agents prior to planned anti-CD19 CAR T-cells is unknown, and should be avoided in the absence of data showing it does not impair the curative potential of subsequent anti-CD19 CAR T-cells.

\section{THE FUTURE OF CAR T}

\section{Expanding eligibility}

Patients with secondary CNS lymphoma were excluded from the initial axi-cel and tisa-cel trials given the theoretical concern for CAR T-cell related neurotoxicity but were eligible for liso-cel in TRANSCEND as long as there was also measurable non-CNS disease. Overall, 7/269 (3\%) of patients in TRANSCEND had 
secondary CNS lymphoma and of the 6 evaluable patients, the ORR and CR rates were both 50\%, compared to rates of $73 \%$ and $53 \%$, respectively, for patients without secondary CNS lymphoma ${ }^{[9]}$. No CNS lymphoma patients had severe CRS, and only $2 / 6$ patients (33\%) experienced neurologic events (both grade 3 ). A retrospective analysis of 8 patients with secondary CNS lymphoma treated with tisa-cel showed a response rate of 50\% (4/8 patients; 2 CR and 2 PR at day 28), and no patients experienced greater than grade 1 neurotoxicity ${ }^{[49]}$. Similar efficacy rates and side effect profiles have been documented in small series of secondary CNS lymphoma patients treated with axi-cel ${ }^{[50-52]}$. These data, albeit with relatively small numbers, provide evidence of anti-tumor activity without significant rates of toxicity and may open new treatment options for patients with historically dismal prognoses.

Building upon the biologic rationale for CAR T-cell activity in secondary CNS lymphoma, studies are ongoing to assess CAR $\mathrm{T}$ in primary CNS lymphoma, including an ongoing prospective pilot trial evaluating the safety and efficacy of tisa-cel (NCT04134117) and an ongoing phase 1 trial of an autologous CD19 specific, hinge-optimized CD28 costimulatory CAR construct generated by the City of Hope (which also includes secondary CNS lymphoma) ${ }^{[53]}$.

Initial CD19 CAR T trials have focused on patients with DLBCL and other relatively common lymphomas. The TRANSCEND trial ${ }^{[9]}$ did include some rarer lymphomas including grade $3 \mathrm{~B} \mathrm{FL}$, lymphomas transformed from indolent histologies other than FL, and secondary CNS lymphoma, and while liso-cel showed activity in a proportion of these patients, the relatively small numbers make subset analyses statistically challenging. In the future, a basket approach combining multiple rare lymphoma subtypes in a single trial may allow insight into CAR T activity in these diseases and potentially lead to expanded CAR Tcell indications.

\section{CAR T "plus" combinations}

An ongoing question in the field is whether combining agents with CAR $\mathrm{T}$ will improve response rates and durations without contributing additional toxicity. Multiple studies are underway to interrogate CAR T in combination with immune checkpoint blockade, including ZUMA-6 (atezolizumab following axi-cel in refractory DLBCL; NCT02926833) ${ }^{[54]}$, PORTIA (pembrolizumab following tisa-cel in relapsed/refractory DLBCL; NCT03630159) $)^{[55]}$, and PLATFORM (durvalumab following liso-cel in relapsed/refractory aggressive B-cell NHL; NCT03310619). PLATFORM is also exploring liso-cel in combination with the immunomodulators CC-122 and CC-220.

Preclinical studies have shown that the BTKi ibrutinib improves CAR T-cell function, quality, and antitumor responses ${ }^{[56,57]}$, suggesting that BTKi in combination with CAR T might lead to improved outcomes. All patients treated with axi-cel in ZUMA-2 $2^{[12]}$ and with liso-cel in the TRANSCEND CLL cohort ${ }^{[43]}$ had prior BTKi exposure by eligibility criteria, as did $88 \%$ of patients treated with liso-cel in the TRANSCEND MCL cohort ${ }^{[25]}$, and some patients were also bridged with BTKi, and thus BTKi exposure might have led to immune system changes that contributed to CAR T responses. Several ongoing studies are addressing BTKi/CAR T combinations, including tisa-cel with ibrutinib in relapsed/refractory DLBCL (NCT03876028), a CD19 CAR T construct with ibrutinib in relapsed/refractory CLL (NCT01865617 ${ }^{[58]}$, and liso-cel with ibrutinib for relapsed/refractory CLL [PLATFORM (NCT03310619) and TRANSCEND (NCT03331198) ${ }^{[59]}$. In a recent interim analysis, liso-cel with ibrutinib led to an ORR of 95\% (18/19 CLL patients) with a CR/CRi in $47 \%$ (9/19) of patients with greater than 1 month of follow up, and relatively low rates of CAR Tassociated toxicities ${ }^{[59]}$. In the trial with a CD19 CAR T construct, the ORR at 1 month was $83 \%(15 / 18$ evaluable CLL patients), with a 1 -year OS of $86 \%$ and PFS of $59 \%$, and a lower severity of CRS in patients treated with CAR T-cells with ibrutinib compared to those patients not given concurrent ibrutinib ${ }^{[58]}$. 


\section{Advances in CAR T design and production}

As highlighted previously, a proportion of patients who undergo apheresis never make it to CAR T infusion, which in addition to progressive disease despite bridging therapy can also be due to CAR T production time and/or manufacturing failures. Clinical trials are ongoing with allogeneic CAR T-cells, which by virtue of being "off the shelf", minimize wait time and may even abrogate the need for bridging and its associated pitfalls. Moreover, allogeneic CAR T approaches circumvent the risk of dysfunctional autologous T-cells arising from exposure to multiple prior therapies and/or primary disease-associated impaired T-cell function ${ }^{[0]}$. ALPHA-2 is an ongoing single arm open label phase $1 / 2$ clinical trial evaluating the safety and efficacy of an allogeneic anti-CD19 CAR T-cell product (ALLO-501A) in relapsed/refractory large B-cell lymphoma (NCT04416984). While allogeneic CARs come with the theoretical risks of rejection and graft versus host disease (GVHD) that are absent with autologous products, gene editing approaches with CRISPR-Cas9, TALEN ${ }^{[61]}$, or ZFN technologies can be used to disrupt HLA and native T-cell receptor gene expression to reduce the risks of rejection and GVHD, respectively. Studies are also ongoing with allogeneic CAR-transduced products generated from NK cells, which have been associated with a lower risk of CRS, neurologic toxicity, and GVHD compared to T-cell derived products. In early results from a phase $1 / 2$ clinical trial (NCT03056339) of HLA-mismatched anti-CD19 CAR-NK cells derived from cord blood administered to 11 patients with relapsed or refractory CD19-positive NHL or CLL, 73\% (8/11) of patients had a response and 7/8 responses were complete, with no instances of CRS, neurologic events, or GVHD ${ }^{[62]}$.

While approximately half of CAR T-treated patients have long term responses, about half of patients do not respond or relapse, underscoring the need for novel CAR T approaches addressing mechanisms of resistance, including target antigen loss, immune escape or silencing, T-cell exhaustion, and loss of persistence. Investigations into antigen escape from lost CD19 expression on lymphoma cells include targeting multiple antigens at once with approaches such as tandem CARs which co-express two targets (e.g., CD19 and CD22 ${ }^{[63]}$ or pools of cells transduced with CARs conferring different antigen specificity. Combinations of CAR T-cells with immune checkpoint blockade and/or immunomodulatory agents as discussed previously may help address immune escape. Moreover, CAR T-cells have been engineered to secrete cytokines such as IL-12 and IL-18 to overcome the immune suppressant tumor microenvironment ${ }^{[6,65]}$ or to directly inhibit immune checkpoints ${ }^{[66]}$. Other novel approaches include third generation CARs which incorporate a co-stimulation domain to enhance T-cell activation ${ }^{[6]}$ or insertion of the CAR gene at the TRAC locus to optimize activation and reduce T-cell exhaustion ${ }^{[68]}$.

\section{CONCLUSION}

CAR T-cells have ushered in a new era of therapeutic options for patients with relapsed/refractory large Bcell, mantle cell, and FLs. Ongoing studies in expanded histologies including relapsed/refractory indolent Bcell lymphomas, and studies addressing optimal CAR T-cell timing and administration in large B-cell lymphomas, may ultimately lead to additional approvals. While significant advances continue to be made in the CAR T-cell space, ongoing research particularly into the biology of CAR T resistance may lead to novel approaches that translate into improved outcomes for lymphoma patients.

\section{DECLARATIONS}

\section{Authors' contributions}

Researched, wrote, edited and reviewed the manuscript: Haydu JE

Wrote, edited and reviewed the manuscript: Abramson JS

\section{Availability of data and materials}

Not applicable. 


\section{Financial support and sponsorship}

None.

\section{Conflict of interest}

Abramson JS reports consulting for Bristol-Myers Squibb, Celgene, Gilead, Kite Pharma, Novartis and Allogene. Haydu JE reports no disclosures.

\section{Ethical approval and consent to participate}

Not applicable.

\section{Consent for publication}

Not applicable.

\section{Copyright}

(C)The Author(s) 2021.

\section{REFERENCES}

1. $\quad$ Siegel RL, Miller KD, Fuchs HE, Jemal A. Cancer Statistics, 2021. CA Cancer J Clin 2021;71:7-33. DOI PubMed

2. Swerdlow SH, Campo E, Pileri SA, et al. The 2016 revision of the World Health Organization classification of lymphoid neoplasms. Blood 2016;127:2375-90. DOI PubMed PMC

3. Crump M, Neelapu SS, Farooq U, et al. Outcomes in refractory diffuse large B-cell lymphoma: results from the international SCHOLAR-1 study. Blood 2017;130:1800-8. DOI PubMed PMC

4. Epperla N, Hamadani M, Cashen AF, et al. Predictive factors and outcomes for ibrutinib therapy in relapsed/refractory mantle cell lymphoma-a "real world" study. Hematol Oncol 2017;35:528-35. DOI PubMed

5. Jain P, Kanagal-Shamanna R, Zhang S, et al. Long-term outcomes and mutation profiling of patients with mantle cell lymphoma (MCL) who discontinued ibrutinib. Br J Haematol 2018;183:578-87. DOI PubMed

6. Martin P, Maddocks K, Leonard JP, et al. Postibrutinib outcomes in patients with mantle cell lymphoma. Blood 2016;127:1559-63. DOI PubMed

7. Casulo C, Byrtek M, Dawson KL, et al. Early relapse of follicular lymphoma after rituximab plus cyclophosphamide, doxorubicin, vincristine, and prednisone defines patients at high risk for death: an analysis from the National LymphoCare Study. J Clin Oncol 2015;33:2516-22. DOI PubMed PMC

8. Jain P, Keating M, Wierda W, et al. Outcomes of patients with chronic lymphocytic leukemia after discontinuing ibrutinib. Blood 2015;125:2062-7. DOI PubMed PMC

9. Abramson JS, Palomba ML, Gordon LI, et al. Lisocabtagene maraleucel for patients with relapsed or refractory large B-cell lymphomas (TRANSCEND NHL 001): a multicentre seamless design study. Lancet 2020;396:839-52. DOI PubMed

10. Neelapu SS, Locke FL, Bartlett NL, et al. Axicabtagene ciloleucel CAR T-cell therapy in refractory large B-cell lymphoma. $N$ Engl $J$ Med 2017;377:2531-44. DOI PubMed PMC

11. Schuster SJ, Bishop MR, Tam CS, et al; JULIET Investigators. Tisagenlecleucel in adult relapsed or refractory diffuse large B-cell lymphoma. N Engl J Med 2019;380:45-56. DOI PubMed

12. Wang M, Munoz J, Goy A, et al. KTE-X19 CAR T-cell therapy in relapsed or refractory mantle-cell lymphoma. $N$ Engl J Med 2020;382:1331-42. DOI PubMed PMC

13. Jacobson C, Chavez JC, Sehgal AR, et al. Primary analysis of Zuma-5: a phase 2 Study of Axicabtagene Ciloleucel (Axi-Cel) in patients with relapsed/refractory (R/R) indolent non-Hodgkin lymphoma (iNHL). Blood 2020;136:40-1.

14. Harding FA, McArthur JG, Gross JA, Raulet DH, Allison JP. CD28-mediated signalling co-stimulates murine T cells and prevents induction of anergy in T-cell clones. Nature 1992;356:607-9. DOI PubMed

15. Savoldo B, Ramos CA, Liu E, et al. CD28 costimulation improves expansion and persistence of chimeric antigen receptor-modified T cells in lymphoma patients. J Clin Invest 2011;121:1822-6. DOI PubMed PMC

16. Salter AI, Ivey RG, Kennedy JJ, et al. Phosphoproteomic analysis of chimeric antigen receptor signaling reveals kinetic and quantitative differences that affect cell function. Sci Signal 2018;11:1-17. DOI PubMed PMC

17. Turtle CJ, Hanafi LA, Berger C, et al. Immunotherapy of non-Hodgkin's lymphoma with a defined ratio of CD8+ and CD4+ CD19specific chimeric antigen receptor-modified T cells. Sci Transl Med 2016;8:1-12. DOI PubMed PMC

18. Locke FL, Ghobadi A, Jacobson CA, et al. Neelapu SS. Long-term safety and activity of axicabtagene ciloleucel in refractory large Bcell lymphoma (ZUMA-1): a single-arm, multicentre, phase 1-2 trial. Lancet Oncol 2019;20:31-42. DOI PubMed PMC

19. Ramsborg CG, Guptill P, Weber C, et al. JCAR017 is a defined composition CAR T cell product with product and process controls that deliver precise doses of CD4 and CD8 CAR T cell to patients with NHL. Blood 2017;130:4471. DOI

20. Sommermeyer D, Hudecek M, Kosasih PL, et al. Chimeric antigen receptor-modified T cells derived from defined CD8+ and CD4+ subsets confer superior antitumor reactivity in vivo. Leukemia 2016;30:492-500. DOI PubMed PMC 
21. Jacobson CA, Hunter BD, Redd R, et al. Axicabtagene Ciloleucel in the non-trial setting: outcomes and correlates of response, resistance, and toxicity. J Clin Oncol 2020;38:3095-106. DOI PubMed PMC

22. Jaglowski S, Hu ZH, Zhang Y, et al. Tisagenlecleucel chimeric antigen receptor (CAR) T-cell therapy for adults with diffuse large Bcell lymphoma (DLBCL): real world experience from the Center for International Blood \& Marrow Transplant Research (CIBMTR) cellular therapy (CT) registry. Blood 2019;134:766. DOI

23. Nastoupil LJ, Jain MD, Feng L, et al. Standard-of-care Axicabtagene Ciloleucel for relapsed or refractory large B-cell lymphoma: results from the US lymphoma CAR T consortium. J Clin Oncol 2020;38:3119-28. DOI PubMed PMC

24. Pasquini MC, Locke FL, Herrera AF, et al. Post-marketing use outcomes of an anti-CD19 chimeric antigen receptor (CAR) T cell therapy, axicabtagene ciloleucel (axi-cel), for the treatment of large B cell lymphoma (LBCL) in the United States (US). Blood 2019;134:764. DOI

25. Palomba ML, Gordon LI, Siddiqi T, et al. Safety and preliminary efficacy in patients with relapsed/refractory mantle cell lymphoma receiving lisocabtagene maraleucel in transcend NHL 001. Blood 2020;136:10-1.

26. Schuster SJ, Svoboda J, Chong EA, et al. Chimeric antigen receptor T cells in refractory B-cell lymphomas. $N$ Engl J Med 2017;377:2545-54. DOI PubMed PMC

27. Hirayama AV, Gauthier J, Hay KA, et al. High rate of durable complete remission in follicular lymphoma after CD19 CAR-T cell immunotherapy. Blood 2019;134:636-40. DOI PubMed PMC

28. Hay KA, Hanafi LA, Li D, et al. Kinetics and biomarkers of severe cytokine release syndrome after CD19 chimeric antigen receptormodified T-cell therapy. Blood 2017;130:2295-306. DOI PubMed PMC

29. Lee DW, Santomasso BD, Locke FL, et al. ASTCT consensus grading for cytokine release syndrome and neurologic toxicity associated with immune effector cells. Biol Blood Marrow Transplant 2019;25:625-38. DOI PubMed

30. Turtle CJ, Hanafi LA, Berger C, et al. CD19 CAR-T cells of defined CD4+:CD8+ composition in adult B cell ALL patients. $J$ Clin Invest 2016;126:2123-38. DOI PubMed PMC

31. Santomasso BD, Park JH, Salloum D, et al. Clinical and biological correlates of neurotoxicity associated with CAR T-cell therapy in patients with B-cell acute lymphoblastic leukemia. Cancer Discov 2018;8:958-71. DOI PubMed PMC

32. Gust J, Hay KA, Hanafi LA, et al. Endothelial activation and blood-brain barrier disruption in neurotoxicity after adoptive immunotherapy with CD19 CAR-T cells. Cancer Discov 2017;7:1404-19. DOI PubMed PMC

33. Nellan A, McCully CML, Cruz Garcia R, et al. Improved CNS exposure to tocilizumab after cerebrospinal fluid compared to intravenous administration in rhesus macaques. Blood 2018;132:662-6. DOI PubMed PMC

34. Brudno JN, Somerville RP, Shi V, et al. Allogeneic T cells that express an anti-CD19 chimeric antigen receptor induce remissions of B-cell malignancies that progress after allogeneic hematopoietic stem-cell transplantation without causing graft-versus-host disease. $J$ Clin Oncol 2016;34:1112-21. DOI PubMed PMC

35. Oluwole OO, Bishop MR, Gisselbrecht C, et al. ZUMA-7: A phase 3 randomized trial of axicabtagene ciloleucel (Axi-Cel) versus standard-of-care (SOC) therapy in patients with relapsed/refractory diffuse large B cell lymphoma (R/R DLBCL). J Clin Oncol 2018;36:TPS7585. DOI

36. Westin J, Bishop M, Flinn I, et al. BELINDA: a phase 3 study evaluating the safety and efficacy of tisagenlecleucel versus standard of care in adult patients with relapsed/refractory aggressive B-cell non-Hodgkin lymphoma. Clin Lymphoma Myeloma Leuk 2019;19:S270-1. DOI

37. Neelapu SS, Dickinson M, Ulrickson ML, et al. Interim analysis of ZUMA-12: a phase 2 study of axicabtagene ciloleucel (axi-cel) as first-line therapy in patients (Pts) with high-risk large B cell lymphoma (LBCL). Blood 2020;136:49.

38. Le Gouill S, Ghesquieres H, Oberic L, et al. Obinutuzumab vs rituximab for advanced DLBCL: a PET-guided and randomized phase 3 study by LYSA. Blood 2021;137:2307-20. DOI PubMed

39. Fowler NH, Dickinson M, Dreyling M, et al. Efficacy and safety of tisagenlecleucel in adult patients with relapsed/refractory follicular lymphoma: interim analysis of the phase 2 Elara trial. Blood 2020;136:1-3.

40. Kochenderfer JN, Dudley ME, Kassim SH, et al. Chemotherapy-refractory diffuse large B-cell lymphoma and indolent B-cell malignancies can be effectively treated with autologous T cells expressing an anti-CD19 chimeric antigen receptor. J Clin Oncol 2015;33:540-9. DOI PubMed PMC

41. Porter DL, Hwang WT, Frey NV, et al. Chimeric antigen receptor T cells persist and induce sustained remissions in relapsed refractory chronic lymphocytic leukemia. Sci Transl Med 2015;7:303ra139. DOI PubMed PMC

42. Turtle CJ, Hay KA, Hanafi LA, et al. Durable molecular remissions in chronic lymphocytic leukemia treated with CD19-specific chimeric antigen receptor-modified T cells after failure of ibrutinib. J Clin Oncol 2017;35:3010-20. DOI PubMed PMC

43. Siddiqi T, Soumerai JD, Dorritie KA, et al. Updated follow-up of patients with relapsed/refractory chronic lymphocytic leukemia/small lymphocytic lymphoma treated with lisocabtagene maraleucel in the phase 1 monotherapy cohort of transcend CLL 004, including high-risk and ibrutinib-treated patients. Blood 2020;136:40-1.

44. Chavez JC, Bachmeier C, Kharfan-Dabaja MA. CAR T-cell therapy for B-cell lymphomas: clinical trial results of available products. Ther Adv Hematol 2019;10:2040620719841581. DOI PubMed PMC

45. Jacobsen ED, Sharman JP, Oki Y, et al. Brentuximab vedotin demonstrates objective responses in a phase 2 study of relapsed/refractory DLBCL with variable CD30 expression. Blood 2015;125:1394-402. DOI PubMed

46. Goy A, Ramchandren R, Ghosh N, et al. Ibrutinib plus lenalidomide and rituximab has promising activity in relapsed/refractory nongerminal center B-cell-like DLBCL. Blood 2019;134:1024-36. DOI PubMed PMC

47. Sehn LH, Herrera AF, Flowers CR, et al. Polatuzumab vedotin in relapsed or refractory diffuse large B-cell lymphoma. J Clin Oncol 2020;38:155-65. DOI PubMed PMC 
48. Salles G, Duell J, Gonzalez Barca E, et al. Tafasitamab plus lenalidomide in relapsed or refractory diffuse large B-cell lymphoma (LMIND): a multicentre, prospective, single-arm, phase 2 study. Lancet Oncol 2020;21:978-88. DOI PubMed

49. Frigault MJ, Dietrich J, Martinez-Lage M, et al. Tisagenlecleucel CAR T-cell therapy in secondary CNS lymphoma. Blood 2019;134:860-6. DOI PubMed PMC

50. Novo M, Ruff MW, Skrabek PJ, Lin Y. Axicabtagene ciloleucel chimeric antigen receptor T Cell therapy in lymphoma with secondary central nervous system involvement. Mayo Clin Proc 2019;94:2361-4. DOI PubMed

51. Bennani NN, Maurer MJ, Nastoupil LJ, et al. Experience with axicabtagene ciloleucel (axi-cel) in patients with secondary CNS involvement: results from the US lymphoma CAR T consortium. Blood 2019;134:763. DOI

52. Abbasi A, Peeke S, Shah N, et al. Axicabtagene ciloleucel CD19 CAR-T cell therapy results in high rates of systemic and neurologic remissions in ten patients with refractory large B cell lymphoma including two with HIV and viral hepatitis. $J$ Hematol Oncol 2020;13:1. DOI PubMed PMC

53. Siddiqi T, Wang X, Palmer J, et al. CD19-targeting CAR-T cell therapy in CNS lymphoma. Blood 2019;134:4075. DOI

54. Jacobson CA, Westin JR, Miklos DB, et al. Abstract CT055: phase 1/2 primary analysis of ZUMA-6: axicabtagene ciloleucel (Axi$\mathrm{Cel}$ ) in combination with atezolizumab (Atezo) for the treatment of patients (Pts) with refractory diffuse large B cell lymphoma (DLBCL). Cancer Res 2020;80:CT055. DOI

55. Jaeger U, Worel N, McGuirk JP, et al. Portia: a phase $1 \mathrm{~b}$ study evaluating safety and efficacy of tisagenlecleucel and pembrolizumab in patients with relapsed/refractory diffuse large B-CELL lymphoma. Blood 2019;134:5325. DOI

56. Ruella M, Kenderian SS, Shestova O, et al. The Addition of the BTK inhibitor ibrutinib to anti-CD19 chimeric antigen receptor T cells (CART19) improves responses against mantle cell lymphoma. Clin Cancer Res 2016;22:2684-96. DOI PubMed

57. Fraietta JA, Beckwith KA, Patel PR, et al. Ibrutinib enhances chimeric antigen receptor T-cell engraftment and efficacy in leukemia. Blood 2016;127:1117-27. DOI PubMed PMC

58. Gauthier J, Hirayama AV, Purushe J, et al. Feasibility and efficacy of CD19-targeted CAR T cells with concurrent ibrutinib for CLL after ibrutinib failure. Blood 2020;135:1650-60. DOI PubMed PMC

59. Wierda WG, Dorritie KA, Munoz J, et al. Transcend CLL 004: phase 1 cohort of lisocabtagene maraleucel (liso-cel) in combination with ibrutinib for patients with relapsed/refractory $(\mathrm{R} / \mathrm{R})$ chronic lymphocytic leukemia/small lymphocytic lymphoma (CLL/SLL). Blood 2020;136:39-40.

60. Fraietta JA, Lacey SF, Orlando EJ, et al. Determinants of response and resistance to CD19 chimeric antigen receptor (CAR) T cell therapy of chronic lymphocytic leukemia. Nat Med 2018;24:563-71. DOI PubMed PMC

61. Qasim W, Zhan H, Samarasinghe S, et al. Molecular remission of infant B-ALL after infusion of universal TALEN gene-edited CAR T cells. Sci Transl Med 2017;9:eaaj2013. DOI PubMed

62. Liu E, Marin D, Banerjee P, et al. Use of CAR-transduced natural killer cells in CD19-positive lymphoid tumors. $N$ Engl J Med 2020;382:545-53. DOI PubMed PMC

63. Fry TJ, Shah NN, Orentas RJ, et al. CD22-targeted CAR T cells induce remission in B-ALL that is naive or resistant to CD19-targeted CAR immunotherapy. Nat Med 2018;24:20-8. DOI PubMed PMC

64. Koneru M, Purdon TJ, Spriggs D, Koneru S, Brentjens RJ. IL-12 secreting tumor-targeted chimeric antigen receptor T cells eradicate ovarian tumors in vivo. Oncoimmunology 2015;4:e994446. DOI PubMed PMC

65. Avanzi MP, Yeku O, Li X, et al. Engineered tumor-targeted T cells mediate enhanced anti-tumor efficacy both directly and through activation of the endogenous immune system. Cell Rep 2018;23:2130-41. DOI PubMed PMC

66. Cherkassky L, Morello A, Villena-Vargas J, et al. Human CAR T cells with cell-intrinsic PD-1 checkpoint blockade resist tumormediated inhibition. J Clin Invest 2016;126:3130-44. DOI PubMed PMC

67. Ramos CA, Rouce R, Robertson CS, et al. In vivo fate and activity of second- versus third-generation CD19-specific CAR-T cells in B cell non-Hodgkin's lymphomas. Mol Ther 2018;26:2727-37. DOI PubMed PMC

68. Eyquem J, Mansilla-Soto J, Giavridis T, et al. Targeting a CAR to the TRAC locus with CRISPR/Cas9 enhances tumour rejection. Nature 2017;543:113-7. DOI PubMed PMC 